\title{
LEBESGUE SETS AND INSERTION OF A CONTINUOUS FUNCTION
}

\author{
ERNEST P. LANE 1
}

\begin{abstract}
Necessary and sufficient conditions in terms of Lebesgue sets are presented for the following two insertion properties for real-valued functions defined on a topological space: (1) If $g \leq f$ there is a continuous function $h$ such that $g \leq h \leq f$, and for each $x$ for which $g(x)<f(x)$ then $g(x)<h(x)<f(x)$.

(2) If $g<f$ there is a continuous function $h$ such that $g<h<f$.
\end{abstract}

1. Statement of results. All functions considered are real-valued. Let $R$ (respectively, $Q$ ) denote the real (respectively, rational) numbers and write $g \leq f$ (respectively, $g<f$ ) in case $g(x) \leq f(x)$ (respectively, $g(x)<f(x)$ ) for each $x$ in the space. For $b$ in $R$ the Lebesgue sets for a function $f$ are defined by $L_{b}(f)=\{x: f(x) \leq b\}$ and $L^{b}(f)=\{x: f(x) \geq b\}$. Let $C^{*}(X)$ denote the lattice of continuous, bounded, and real-valued functions on $X$. The main results of this paper utilize Lebesgue sets to characterize certain insertion properties of a continuous function, and they are based on the following ([2, Theorem $3.5 ; 8$, Theorem 2.1]):

THEOREM 1. If $g \leq f$ then there is a continuous function $h$ such that $g \leq h \leq f$ if and only if for any rational numbers $a$ and $b$ such that $a<b$ the Lebesgue sets $L^{b}(g)$ and $L_{a}(f)$ are completely separated.

The following is stated on p. 444 of [11].

THEOREM 2. Let $X$ be a topological space and let $L(X)$ and $U(X)$ be classes of bounded functions defined on $X$ such that any constant function is in $L(X) \cap U(X)$ and such that if $g \in U(X), f \in L(X)$, and $r \in R$ then $g \wedge r \in U(X)$ and $f \vee r \in L(X)$. The following are equivalent:

(i) If $f \in L(X), g \in U(X)$, and $g \leq f$ there exists $h$ in $C^{*}(X)$ such that $g \leq h \leq f$ and such that $g(x)<h(x)<f(x)$ for each $x$ for which $g(x)<f(x)$.

(ii) If $f \in L(X), g \in U(X)$, and $r \in R$ the Lebesgue sets $L_{r}(f)$ and $L^{r}(g)$ are zero sets in $X$.

(iii) If $f \in L(X)$ and $g \in U(X)$ then $f$ (respectively, $g$ ) is the pointwise limit of and increasing (respectively, decreasing) sequence of continuous functions.

In the situation where $U(X)$ and $L(X)$ are the classes of upper and lower semicontinuous functions, respectively, the equivalence of (i) and (ii) of the above theorem is due to Michael [12], the equivalence of (ii) and (iii) is due to Tong [14], and each of the conditions being equivalent to $X$ is perfectly normal. If $U(X)$ and $L(X)$ are the classes of normal upper and normal lower semicontinuous functions,

Received by the editors August 28, 1981 and, in revised form, November 10, 1981; presented to the Society, August 1981.

1980 Mathematics Subject Classification. Primary 54C30; Secondary 54C05, 54C50.

Key words and phrases. Insertion of continuous functions, Lebesgue set, completely separated.

${ }^{1}$ This research was supported by a released teaching load research grant from Appalachian State University.

(c) 1983 American Mathematical Society 0002-9939/81/0000-0287/\$02.25 
respectively, the equivalence of (i), (ii), (iii), and $X$ is an $\mathrm{Oz}$ space is established in [8].

Necessary and sufficient conditions in order for a space to satisfy condition (i) of Theorem 2 for general classes of functions are considered in $[12,3,4,7$, and 10]. Let $B(X)$ denote the Banach lattice of all bounded real-valued functions on a space $X$. If $C$ is a sublattice of the power set of $X$ to which $\varnothing$ and $X$ belong, the smallest convex cone in $B(X)$ that contains the constant functions $1_{D}, D \in C$, is denoted by $c n(C)$ and its closure by $\overline{c n}(C)$. The results of Blatter and Seever in [3 and 4] require that the classes $U(X)$ and $L(X)$ can be characterized as $\overline{c n}(A)$ and $\overline{c n}(B)$, respectively, for some sublattices $A$ and $B$ of the power set of $X$ and that $A \subset B_{\delta}$ (= intersection of sequences in $B$ ) and $B \subset A_{\sigma}$ (= union of sequences in $A$ ). The necessary (as proved by Powderly [13]) and sufficient condition of [7] places restrictions on the function $f-g$. These limitations are avoided in Theorem 2.

A portion of the following result is stated on p. 478 of [11].

THEOREM 3. Let $L(X)$ and $U(X)$ be classes of bounded real-valued functions on $X$ such that $C^{*}(X) \subset L(X) \cap U(X)$. The following are equivalent:

(i) For $f \in L(X), g \in U(X)$ and $g<f$ there exists $h \in C^{*}(X)$ such that $g<h<f$.

(ii) If $f \in L(X), g \in U(X)$ and $g<f$ then for each $r$ in $Q$ there exist disjoint sets $A_{r}$ and $B_{r}$ such that $L_{r}(f)$ and $X-A_{r}$ are completely separated, $L^{r}(g)$ and $X-B_{r}$ are completely separated, and each of $\left\{X-\left(B_{r} \cup L_{r}(f)\right): r \in Q\right\}$ and $\left\{X-\left(A_{r} \cup L^{r}(g)\right): r \in Q\right\}$ covers $X$.

(iii) If $f \in L(X), g \in U(X)$ and $g<f$ then for each rational number $r$ there exist disjoint sets $A_{r}$ and $B_{r}$ such that $L_{r}(f)$ and $X-A_{r}$ are completely separated, $L^{r}(g)$ and $X-B_{r}$ are completely separated, and $\left\{X-\left(A_{r} \cup B_{r}\right): r \in Q\right\}$ covers $X$.

In the situation in which $L(X)$ and $U(X)$ are the lattices of lower and upper semicontinuous functions, respectively, results of Dowker [5] and Katětov [6] show that a space satisfies (i) of the above theorem if and only if $X$ is normal and countably paracompact. Other specific cases are given in [10, Theorem 4.2]. Necessary and sufficient conditions for a space to satisfy (i) of Theorem 3 for general classes of functions are given in $[3,4$, and 7$]$ but these results have restrictions analogous to those mentioned in the discussion following Theorem 2.

It is noted that the bounded condition placed on the functions in Theorems 2 and 3 causes no loss in generality if the properties that define the classes $L(X)$ and $U(X)$ are preserved under an order preserving homeomorphism from $R$ onto a finite interval.

2. Proofs of results. The following lemma is used in combination with Theorem 1 in proving Theorems 2 and 3 . The argument is adapted from a technique used in the proof of Theorem 3.3 in [3] and is included here for completeness.

LEMMA 1. Let $f, g$ and $k$ be bounded functions such that $g \leq k \leq f$ and $k \in$ $C^{*}(X)$. If there exist sequences $\left\{a_{n}\right\}$ and $\left\{b_{n}\right\}$ in $C^{*}(X)$ such that $g \leq a_{n}$ and $b_{n} \leq f$

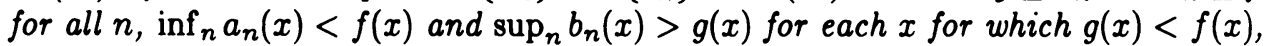
then there exists $h$ in $C^{*}(X)$ such that $g \leq h \leq f$ and for each $x$ for which $g(x)<f(x)$ then $g(x)<h(x)<f(x)$. 
PROOF. Using the notation of the lemma, set

$$
h=\sum_{n=1}^{\infty} 2^{-n-1}\left(a_{n} \wedge k+b_{n} \wedge k\right) .
$$

Since $g \leq a_{n} \wedge k, \sum_{n=1}^{\infty} 2^{-n} g \leq \sum_{n=1}^{\infty} 2^{-n}\left(a_{n} \wedge k\right)$, or $g \leq \sum_{n=1}^{\infty} 2^{-n}\left(a_{n} \wedge k\right)$. Similarly, from $f \geq a_{n} \wedge k$ it follows that $f \geq \sum_{n=1}^{\infty} 2^{-n}\left(a_{n} \wedge k\right)$. Thus $g \leq$ $\sum_{n=1}^{\infty} 2^{-n}\left(a_{n} \wedge k\right) \leq f$. In the same fashion show that $g \leq \sum_{n=1}^{\infty} 2^{-n}\left(k \vee b_{n}\right) \leq f$. From the definition of $h$ it follows that $g \leq h \leq f$. Let $x$ be such that $g(x)<f(x)$. Choose $N$ so that $a_{N}(x)<f(x)$. Then $\sum_{n=1}^{\infty} 2^{-n}\left(a_{n} \wedge k\right)(x)=\sum_{n \neq N} 2^{-n}\left(a_{n} \wedge\right.$ $k)(x)+2^{-N}\left(a_{N} \wedge k\right)(x)<\sum_{n \neq N} 2^{-n}\left(a_{n} \wedge k\right)(x)+2^{-N} f(x) \leq \sum_{n=1}^{\infty} 2^{-n} f(x)=f(x)$. Similarly, show that $g(x)<\sum_{n=1}^{\infty} 2^{-n}\left(b_{n} \vee k\right)(x)$. Hence $g(x)<h(x)<f(x)$ whenever $g(x)<f(x)$.

That condition (ii) of Theorem 2 implies (i) is a consequence of Proposition 6.1 of [3]. A proof is given here that uses the above lemma since this approach seems considerably more direct.

PROOF OF THEOREM 2. (i) $\Rightarrow$ (ii) If $g \in U(X)$ and $r \in R$ then by hypothesis $g \wedge r \in U(X)$ and $r \in L(X)$. By (i) there is a continuous function $h$ such that $g \wedge r \leq h \leq r$ and if $g(x)<r$ then $g(x)<h(x)<r ; L^{r}(g)$ is a zero set since $L^{r}(g)=$ $\{x: h(x)=r\}$. Similarly show that $L_{r}(f)$ is a zero set.

(ii) $\Rightarrow$ (iii) If $f$ is a lower semicontinuous function defined on a perfectly normal space, Tong's proof [14] that $f$ is a pointwise limit of an increasing sequence of continuous functions is based on the Lebesgue set $L_{r}(f)$ being a zero set; with trivial modification his proof yields this implication.

(iii) $\Rightarrow\left(\right.$ i) Let $g \in U(X)$ and $f \in L(X)$ with $g \leq f$. If $\left\{f_{n}\right\}$ is an increasing sequence of continuous functions whose pointwise limit is $f$ then for any real number $r$ the Lebesgue set $L_{r}(f)$ equals the intersection of the sequence $\left\{L_{r+1 / n}\left(f_{n}\right)\right\}$ of zero sets, and thus $L_{r}(f)$ is a zero set. Similarly, use a decreasing sequence $\left\{g_{n}\right\}$ of continuous functions whose pointwise limit is $g$ to show that each $L^{r}(g)$ is a zero set. For any rational numbers $a<b, L^{b}(g)$ and $L_{a}(f)$ are disjoint zero sets and hence are completely separated; by Theorem 1 there is a continuous function $k$ such that $g \leq k \leq f$. Since the sequences $\left\{g_{n}\right\}$ and $\left\{f_{n}\right\}$ satisfy the conditions of Lemma 1, it follows that there exists $h$ in $C^{*}(X)$ such that $g \leq h \leq f$ and whenever $g(x)<f(x)$ then $g(x)<h(x)<f(x)$. This concludes the proof of Theorem 2 .

If $k$ maps a space $X$ into $R$, call $k$ regular lower semicontinuous (respectively, regular upper semicontinuous) if for each real number $r$ the Lebesgue set $L_{r}(k)$ (respectively, $L^{r}(k)$ ) is a regular $G_{\delta}$ subset of $X$. (These functions were considered in [9].) Let $L(X)$ (respectively, $U(X)$ ) denote the class of bounded regular lower (respectively, upper) semicontinuous functions. The following is an immediate corollary of Theorem 2. If $g \in U(X), f \in L(X)$, and $g \leq f$ there is $h$ in $C^{*}(X)$ such that $g \leq h \leq f$ and such that $g(x)<h(x)<f(x)$ whenever $g(x)<f(x)$ if and only if each regular $G_{\delta}$ subset of $X$ is a zero set. (If $X$ is an Oz space [1] or if $X$ is almost normal then each regular $G_{\delta}$ subset of $X$ is a zero set.)

Proof of TheOREM 3. (i) $\Rightarrow$ (iii) If $f \in L(X), g \in U(X)$ and $g<f$ then by (i) there exists $h$ in $C^{*}(X)$ such that $g<h<f$. Since $C^{*}(X) \subset L(X) \cap U(X)$ we may use hypothesis (i) again to show there exist $h_{1}$ and $h_{2}$ in $C^{*}(X)$ such that $g<$ $h_{1}<h<h_{2}<f$. For each $r$ in $Q$ let $A_{r}=\left\{x: h_{2}(x)<r\right\}$ and $B_{r}=\left\{x: h_{1}(x)>r\right\}$. In order to see that $L_{r}(f)$ and $X-A_{r}$ are completely separated, use (i) to choose $k$ 
in $C^{*}(X)$ such that $h_{2}<k<f$. Since $X-A_{r}$ and $L_{r}(k)$ are disjoint zero sets and $L_{r}(k) \supset L_{r}(f)$ it follows that $L_{r}(f)$ and $X-A_{r}$ are completely separated. Similarly, $L^{r}(g)$ and $X-B_{r}$ are completely separated. The sets $X-\left(A_{r} \cup B_{r}\right), r \in Q$, cover $X$ since $h_{1}<h_{2}$. Thus (iii) is satisfied.

That (iii) implies (ii) is manifest; the argument to show (ii) implies (i) follows: Let $g \in U(X), f \in L(X)$ and suppose that $-M<g<f<M$. It follows from (ii) that for any rational number $r$ that $L_{r}(f)$ and $L^{r}(g)$ are completely separated; in particular for any rationals $a<b$ then $L_{a}(f)$ and $L^{b}(g)$ are completely separated. By Theorem 1 there is $k$ in $C^{*}(X)$ such that $g \leq k \leq f$. For each rational number $r$ choose sets $A_{r}$ and $B_{r}$ satisfying the conditions of (ii), and then choose $a_{r}$ and $b_{r}$ in $C^{*}(X)$ such that $-M \leq a_{r} \leq r, a_{r}=-M$ on $L_{r}(f), a_{r}=r$ on $X-A_{r}, r \leq b_{r} \leq M$, $b_{r}=M$ on $L^{r}(g)$, and $b_{r}=r$ on $X-B_{r}$. If $x \in L_{r}(f)$ then $a_{r}(x)=-M \leq f(x)$ and if $x$ is not in $L_{r}(f)$ then $f(x)>r \geq a_{r}(x)$; thus $a_{r} \leq f$. Let $x \in X$ and choose $s \in Q$ such that $x$ is $X-\left(A_{s} \cup L^{s}(g)\right)$; since $x \in X-L^{s}(g)$ then $g(x)<s$ and since $x \in X-A_{s}$ then $a_{s}(x)=s$. Thus $\sup _{r} a_{r}(x) \geq a_{s}(x)>g(x)$. Similarly, show that $g \leq b_{r}$ for each $r$ and $\inf _{r} b_{r}(x)<f(x)$ for each $x$. By Lemma 1 there exists $h$ in $C^{*}(X)$ such that $g<h<f$. This concludes the proof of Theorem 3 .

As one application of Theorem 3 consider the result of Dowker and Katétov mentioned above. Suppose that $X$ is normal and countably paracompact, $g$ is upper semicontinuous, $f$ is lower semicontinuous and $g<f$. Since $\left\{X-\left(L_{r}(f) \cup\right.\right.$ $\left.\left.L^{r}(g)\right): r \in Q\right\}$ is a countable open cover of $X$ there is a cover $\left\{F_{r}: r \in Q\right\}$ of $X$ such that $F_{r}$ is closed and $F_{r} \subset X-\left(L_{r}(f) \cup L^{r}(g)\right)$ for each $r$. Since $F_{r}$ and $L_{r}(f)$ are completely separated choose $k_{r}$ in $C^{*}(X)$ such that $k_{r}=0$ on $L_{r}(f), k_{r}=1$ on $F_{r}$, and let $A_{r}=\left\{x: k_{r}(x)<\frac{1}{2}\right\}$. Thus $L_{r}(f)$ and $X-A_{r}$ are completely separated. Similarly, define $B_{r}$ so that $L^{r}(g)$ and $X-B_{r}$ are completely separated. Since $F_{r} \subset X-\left(A_{r} \cup B_{r}\right),\left\{X-\left(A_{r} \cup B_{r}\right): r \in Q\right\}$ covers $X$. By Theorem 3 there is $h$ in $C^{*}(X)$ such that $g<h<f$.

\section{REFERENCES}

1. R. L. Blair, Spaces in which special sets are $z$-embedded, Canad. J. Math. 28 (1976), 673-690.

2. _- Extensions of Lebesgue sets and of real-valued functions, Czechoslovak Math. J. 31 (1981), 63-74.

3. J. Blatter and G. L. Seever, Interposition of semicontinuous functions by continuous functions, Analyse Fonctionelle et Applications (Comptes Rendus du Colloque d'Analyse, Rio de Janeiro-1972), Hermann, Paris, 1975, pp. 27-51.

4. - Interposition and lattice cones of functions, Trans. Amer. Math. Soc. 222 (1976), 65-96.

5. C. H. Dowker, On countably paracompact spaces, Canad. J. Math. 3 (1951), 219-224.

6. M. Katêtov, On real-valued functions in topological spaces, Fund. Math. 38 (1951), 85-91.

7. E. P. Lane, Insertion of a continuous function, Pacific J. Math. 66 (1976), 181-190.

8. - PM-normality and the insertion of a continuous function, Pacific J. Math. 82 (1979), 155162.

9. - Weak $C$ insertion of a continuous function, Notices Amer. Math. Soc. 26 (1979), A-231.

10. - Insertion of a continuous function, Topology Proc. 4 (1979), 463-478.

11. - Insertion of a continuous function, Abstracts Amer. Math. Soc. 2 (1981), 788-54-6, 81T54-389.

12. E. Michael, Continuous selections. I, Ann. of Math. 63 (1956), 361-382.

13. M. Powderly, On insertion of a continuous function, Proc. Amer. Math. Soc. 81 (1981), 119-120.

14. H. Tong, Some characterizations of normal and perfectly normal spaces, Duke Math. J. 19 (1952), 289-292.

Department of Mathematics, Appalachian State University, Boone, North CAROLINA 28608 